\title{
A CONTRIBUIÇÃO DO SISTEMA ISO 9000 PARA O DESEMPENHO ESTRATÉGICO: UM ESTUDO EM EMPRESAS BRASILEIRAS DO SETOR QUÍMICO
}

\section{THE CONTRIBUTION GRADE OF THE ISO 9000 QUALITY SYSTEM TO THE STRATEGIC PERFORMANCE OF BRAZILIAN FIRMS IN THE CHEMISTRY SECTOR}

\author{
Célio Gurgel Amorim ${ }^{1}$; Rubens Eugênio Barreto Ramos ${ }^{2}$; Mario Orestes Aguirre González ${ }^{3}$ \\ ${ }^{1}$ Universidade Federal do Rio Grande do Norte - UFRN - Natal - Brasil celiogurgel@digizap.com.br \\ ${ }^{2}$ Universidade Federal do Rio Grande do Norte - UFRN - Natal - Brasil rubens@ct.ufrn.br \\ ${ }^{3}$ Universidade Federal de São Carlos - UFSCar - SP - Brasil mario gonzalez pe@yahoo.com
}

\begin{abstract}
Resumo
O objetivo deste trabalho é analisar o grau de contribuição do sistema de gestão da qualidade, conforme a norma ISO 9001:2000, para o desempenho estratégico das empresas brasileiras do setor químico, tendo como referência o sistema de medição e gestão do desempenho Balanced Scorecard. É desenvolvida uma pesquisa bibliográfica para levantamento das informações técnicas e cientificas existentes sobre os dois temas centrais desta pesquisa - ISO 9000 e Balanced Scorecard, com ênfase na integração dos mesmos. É procedida uma pesquisa de campo do tipo survey, para conhecimento, quantificação e levantamento das informações necessárias para as análises empreendidas. Foram consultadas 352 empresas certificadas do setor químico brasileiro, das quais 111 responderam ao questionário corretamente, o que representa mais de $31 \%$ da população pesquisada. Os principais resultados alcançados mostram visões congruentes dos dirigentes e gerentes na escolha das variáveis significativas que contribuem diretamente para o desempenho estratégico das empresas. Para as empresas analisadas, o sistema ISO 9000 proporciona um grau de contribuição grande para o desempenho estratégico.
\end{abstract}

Palavras-chave: qualidade, desempenho, ISO 9000, balanced scorecard, estratégia.

\section{Introdução}

Nas últimas décadas, um número crescente de empresas no mundo vem adotando sistemas de gestão, baseados em normas que são aceitas internacionalmente, com objetivos de propiciar melhores condições competitivas e garantir a sua permanência no mercado (RENZI \& CAPPELLI, 2000). Como exemplos desta tendência, estão sendo implantadas as normas da série ISO 9000 para a gestão da qualidade, a norma ISO 14001 para a gestão ambiental e as normas BS 8800 ou OHSAS 18000 para a gestão da saúde e segurança do trabalho. 
Outros sistemas têm sido implantados nas organizações com o propósito de melhorar o desempenho estratégico. Dentre estes, os sistemas de gestão do desempenho foram criados para assessorar os gestores nos processos de implementação das estratégias e de tomada de decisão. Um dos sistemas de medição e gestão do desempenho mais conhecidos e pesquisados denomina-se Balanced Scorecard, desenvolvido pelos professores Kaplan e Norton (1997), no início da década de 1990.

Normalmente, estes sistemas são implantados para que as empresas melhorem o desempenho estratégico continuamente. Em contrapartida, são exigidos esforços, dedicação e alocação de recursos para acompanhar e controlar os requisitos normativos, além de uma significativa participação gerencial e técnica nas diversas auditorias periódicas de avaliação destes sistemas, no controle da documentação e na própria operacionalização dos sistemas. Para Brandstetter et al. (2002), as etapas, o tempo para implantação e os custos envolvidos neste processo variam entre as organizações e os diversos segmentos de mercado.

As organizações que implantam o sistema ISO 9000 questionam se é realmente efetiva a sua implementação, ou seja, se existem ganhos estratégicos para as empresas (SUN, 2000). De acordo com a International Organization for Standardization, mais de 550 mil certificados ISO 9000 foram emitidos em 149 países ao final de 2002. Para a Associação Brasileira de Normas Técnicas (ABNT, 2004), no Brasil existiam mais de 5000 certificados emitidos pela nova versão da ISO 9001:2000, com 352 delas no setor químico, ao final do ano de 2003.

\section{Revisão da literatura}

Um sistema da qualidade é definido como a estrutura organizacional, as responsabilidades, os procedimentos, os processos e os recursos para implementar a administração da qualidade. A série ISO 9000 forma um conjunto de procedimentos, padrões e requisitos para funcionamento dos sistemas de gestão da qualidade. Cada país possui seus próprios padrões, baseados na série ISO 9000. No Brasil, as normas da série ISO 9000 são editadas pela Associação Brasileira de Normas Técnicas (ABNT, 2000). O foco desta pesquisa recai sobre a norma ISO 9001:2000, pois é a norma da série cuja certificação é acreditada e reconhecida pela ISO a partir de dezembro de 2003. Para esta norma, a satisfação dos clientes é alcançada através do exercício da liderança, estabelecida nos requisitos referentes à responsabilidade da direção e pela gestão eficaz dos recursos e processos, descritos na norma através dos itens relativos à realização do produto, medição, análise e melhoria.

Por outro lado, Kaplan e Norton (1997) colocam que as empresas na era da informação estão baseadas em um novo conjunto de premissas operacionais, como: processos interfuncionais, ligação com clientes e fornecedores, segmentação dos clientes, escala global, inovação e trabalhadores do 
conhecimento. À medida que as organizações investem na aquisição dessas novas capacidades, o sucesso ou o fracasso não pode mais ser monitorado pelo modelo tradicional da contabilidade financeira, que mede acontecimentos passados, mas por sistemas que explicitem a capacidade de produzir valor no futuro. O sistema Balanced Scorecard integra as medidas derivadas das estratégias e incorpora variáveis com visões de médio e longo prazo, inclusive financeiras e não-financeiras. $\mathrm{O}$ desempenho estratégico pode ser inferido a partir de quatro perspectivas, interligadas através da relação causa e efeito, quais sejam: crescimento e aprendizado; processos internos; mercados e clientes e perspectiva financeira. São selecionados cinco componentes representativos para cada perspectiva, que sejam amplamente utilizados pelas empresas, baseados em diversos trabalhos empreendidos por Kaplan e Norton.

Alguns pesquisadores desenvolveram trabalhos sobre o potencial de integração entre os sistemas da qualidade e do desempenho, ou mais especificamente, sobre o grau de contribuição dos sistemas da qualidade TQM e/ou ISO 9000 no desempenho das empresas. No exterior, citam-se Terziovski et al. (1997); Sun (2000); Zhang (2000); Rahman (2001); Heras et al. (2002); Wayhan et al. (2002); Terziovski et al. (2003); Lorente \& Costa (2003); Conca et al. (2004); Martínez-Lorente \& Martinez-Costa (2004); Martínez-Costa \& Martínez-Lorente, 2004; e Dimara et al. (2004). No Brasil, são divulgados os trabalhos de Leite e Prancic (2003); Schnorr \& Kronmeyer Filho (2003); Menezes \& Martins (2001); Cavalcanti et al. (2004); Hoque (2003); Brandstetter et al. (2002); Coltro (1998); Cerqueira e Moraes (2003); e Lima et al. (2000).

As pesquisas que correlacionam os sistemas de gestão da qualidade e do desempenho ainda são escassas. Existem alguns trabalhos que integram o sistema de gestão da qualidade (TQM) com o desempenho. Outros pesquisadores correlacionam especificamente o sistema ISO 9000 com o desempenho de uma forma geral. Ainda são raros os trabalhos que integram diretamente os sistemas ISO 9000 e BSC. Da análise dos trabalhos publicados, verifica-se que há abordagens distintas e conclusões diversificadas sobre os reais benefícios da certificação. Alguns autores concluem que o sistema ISO 9000 não apresenta impacto positivo no desempenho organizacional (TERZIOVSKI et al. (1997); TERZIOVSKI \& SAMSON (1999); LIMA et al. (2000); SINGEL et al. (2001) e RAHMAN (2001)). Outros pesquisadores demonstraram que o sistema ISO 9000 apresenta uma relação parcial com o desempenho (SUN (2000) e WAYHAN et al. (2002)) e por fim, outros autores mostraram que este sistema contribui de forma positiva para o desempenho estratégico (HERAS et al. (2002) e CHOW-CHUA et al. (2003)). 


\section{Método de pesquisa}

É procedida uma pesquisa quantitativa de campo, através de questionário, para conhecimento e levantamento das informações necessárias para elaboração das análises do desempenho das empresas brasileiras do setor químico que adotaram o sistema ISO 9001:2000. Para aplicação, foi escolhido o setor químico por ser competitivo e ter um número representativo de empresas certificadas por essa norma no Brasil. De acordo com a Associação Brasileira de Normas Técnicas (ABNT, 2004), esse setor possui 352 empresas certificadas. São cadastradas empresas de portes variados, desde pequenas empresas como uma farmácia até grandes conglomerados, como Petrobras, Petroflex, Rhodia e outras mais. Os questionários foram enviados para a população de 352 empresas. A análise estatística foi realizada considerando-se o número real de questionários que retornaram preenchidos corretamente, de forma espontânea. A amostra é composta de 111 empresas, ou $32 \%$ da população.

Vários autores apresentaram suas pesquisas com o emprego de questionários específicos para levantamento das informações, como por exemplo, Zhang (2000), Martinez-Costa e MartinezLorente (2004), Sun (2000) e Rahman (2000). Cada pesquisador seleciona um conjunto de variáveis de acordo com o objeto da análise. As variáveis selecionadas nesta pesquisa para análise do desempenho estratégico do sistema da qualidade ISO 9000 são selecionadas com base no mapa estratégico do Balanced Scorecard padrão, proposto por Kaplan e Norton (1997). As empresas informaram o grau de contribuição do sistema ISO 9000 no desempenho das variáveis selecionadas, comparando-se o desempenho antes e após a certificação.

Para operacionalização das hipóteses de pesquisa foram selecionadas as variáveis descritas na Tabela 1. Para a perspectiva do aprendizado e crescimento, são solicitadas informações sobre a contribuição do sistema ISO 9001:2000 na capacitação e desenvolvimento dos empregados, na tecnologia e disponibilidade das informações, na cultura organizacional, no trabalho em equipe e na liderança. Para a perspectiva dos processos internos, as perguntas elaboradas versam sobre como a certificação influencia os processos internos, os processos de seleção e retenção de clientes, os processos regulatórios e sociais, os processos de inovação e desenvolvimento e os processos de apoio e logísticos. As implicações práticas da norma ISO 9001 no desempenho da perspectiva dos clientes são analisadas através das questões sobre a satisfação dos clientes com preço, qualidade, disponibilidade, serviços e marca. Por fim, a perspectiva financeira é balizada pelas receitas de vendas, custos operacionais, utilização dos ativos, geração de valor para os clientes e retorno sobre o capital empregado. Ao final da seção, são solicitadas informações sobre o grau de contribuição do sistema ISO 9000 no desempenho estratégico, comparando-se o período antes e pós-certificação. As hipóteses nulas e alternativas a serem testadas para cada componente selecionado, detalhadas na 
Tabela 1, são Ho: $\mu \leq 7$ e Ha: $\mu>7$, respectivamente. O parâmetro sete foi selecionado, pois de acordo com Johnson \& Gustafsson (2000), nas pesquisas de onde se avaliam a qualidade e a satisfação dos clientes, na escala de 0 a 10, a resposta normal esperada para este tipo de pesquisa é sete.

Tabela 1 - Discriminação das variáveis

\begin{tabular}{lll}
\hline \multicolumn{1}{c}{ Perspectiva } & Variáveis & Discriminação das variáveis \\
\hline Aprendizado e Crescimento & A1 & Capacitação e desenvolvimento dos empregados \\
A2 & Tecnologia e disponibilidade das informações \\
A3 & Cultura organizacional \\
A4 & Trabalho em equipe \\
A5 & Liderança \\
Perspectiva interna & P1 & Processos de produção \\
& P2 & Processos de conquista e retenção de clientes \\
P3 & Processos de inovação e desenvolvimento \\
P4 & Processos regulatórios e sociais \\
Perspectiva dos clientes & P5 & Processos de apoio e logísticos \\
& S1 & Satisfação dos clientes com o preço \\
& S2 & Satisfação dos clientes com a qualidade \\
S3 & Satisfação dos clientes com a disponibilidade \\
S4 & Satisfação dos clientes com os serviços \\
& S5 & Satisfação dos clientes com a marca \\
& F1 & Receita de venda dos produtos e serviços \\
& F2 & Custos operacionais \\
& F3 & Utilização dos ativos da empresa \\
& F4 & Retorno sobre o Capital empregado (ROCE) \\
& F5 & Valor econômico para os clientes \\
\hline
\end{tabular}

É realizada uma análise descritiva dos dados obtidos com o objetivo de mostrar e conhecer em detalhes as informações obtidas com o questionário aplicado. Em seguida, são realizados os testes de hipóteses, para se verificar se as suposições levantadas são verdadeiras, adotando-se a distribuição t com um nível de significância estatística de 5\%. Para a análise de regressão, foram considerados os 111 respondentes na avaliação das perspectivas aprendizado e crescimento e interna. Entretanto, para as perspectivas clientes e financeira, foram consideradas somente as opiniões dos 30 dirigentes e 51 gerentes, por concentrarem-se nestes níveis gerenciais as informações estratégicas correspondentes, como balanços financeiros e relatórios gerenciais de desempenho.

\section{Resultados e discussão}

A pesquisa foi direcionada para ser respondida pelos dirigentes, que possuem uma visão mais ampla de todos os resultados das empresas. Entretanto, alguns questionários foram respondidos por técnicos $(3 \%)$, profissionais $(9 \%)$ e supervisores $(17 \%)$. Para efeito de verificação das variáveis mais significativas que impactam no desempenho estratégico, optou-se por direcionar a análise das perspectivas cliente e financeira somente às categorias de dirigentes e gerentes. 
Dos 352 questionários enviados às empresas certificadas do setor químico, foram devolvidos 10 questionários por deficiência no endereçamento postal. Algumas questões foram deixadas em branco. As variáveis com maior número de respostas em branco foram concentradas na perspectiva financeira: Retorno sobre o Capital Empregado - ROCE (12), Valor econômico para os clientes (14) e Receitas de vendas (9). Provavelmente, isto demonstra que algumas empresas ainda apresentam algum tipo de restrição a divulgarem os seus resultados econômico-financeiros ou os respondentes desconhecem parcialmente os conceitos ou valores correspondentes. Entretanto, este número de respostas em branco está compatível com pesquisas similares. Conseqüentemente, todos os questionários respondidos foram considerados para as análises empreendidas.

A confiabilidade das medições relativas às variáveis selecionadas pode ser inferida através do coeficiente Alfa de Cronbach. De acordo com Hair et al. (2003), este coeficiente é aceitável até um limite de 0,70. Na pesquisa realizada foram obtidos os seguintes valores: 0,816 para a perspectiva aprendizado e crescimento; 0,826 para a perspectiva interna; 0,878 para a perspectiva clientes e 0,907 para a perspectiva financeira. Por conseguinte, estes valores são superiores ao limite mínimo aceitável, demonstrando a validade das variáveis selecionadas.

Os dados coletados mostram o perfil dos respondentes, onde se destaca o percentual de $89 \%$ de pessoas ligadas diretamente à gestão da empresa estudada, composta de dirigentes, gerentes e supervisores, sendo que $74 \%$ são dirigentes ou gerentes. A função do respondente na empresa desempenha um papel fundamental na qualidade e na representatividade da resposta. Para o tempo de experiência dos respondentes nas empresas analisadas, destacam-se os respondentes com tempo de casa nas faixas compreendidas entre três a seis anos e entre onze e vinte anos de trabalho, que perfazem $62 \%$ do total. As empresas analisadas são relativamente novas, já que $65 \%$ delas foram fundadas após 1970 e 23\% entre 1951 e 1970. As empresas mais maduras com mais de cinqüenta anos de fundação representam apenas $12 \%$ da amostra analisada. Grande parte das empresas não adota ou desconhece o sistema de gestão de desempenho Balanced Scorecard (77\%). A maioria das empresas analisadas é genuinamente brasileira (76\%) e somente $11 \%$ possuem ações negociadas na Bolsa de Valores. Das 111 empresas estudadas, 87\% possuem menos de 500 empregados e 69 são de pequeno porte com menos de 100 empregados.

O construto que representa a perspectiva de aprendizado e crescimento foi composto de cinco variáveis: capacitação e desenvolvimento dos empregados; tecnologia e disponibilidade das informações; cultura organizacional; trabalho em equipe e liderança. As médias obtidas para todos os componentes superam estatisticamente a média 7,0. Ou seja, o grau de contribuição do sistema ISO 9000 no desempenho dos componentes da perspectiva de aprendizado e crescimento é grande.

A contribuição do sistema ISO 9000 se comportou de maneira heterogênea no âmbito da perspectiva interna. Para os componentes relativos aos processos de produção e de conquista e 
retenção de clientes as médias obtidas ficaram acima de sete. Entretanto, para os processos regulatórios e sociais, o desempenho ficou abaixo de sete. Este resultado era esperado devido aos processos regulatórios serem considerados por outros instrumentos normativos. Para os outros dois componentes selecionados não houve rejeição da hipótese nula. Para Benner \& Tushman (2003), algumas empresas que adotam sistemas de gestão da qualidade apresentam desvantagens competitivas em inovação, principalmente devido ao processo de padronização.

Com exceção do componente referente à satisfação dos clientes com o preço, o grau de contribuição do sistema ISO 9000 para o desempenho da perspectiva do cliente é significativo. Esse sistema influencia com um grau de contribuição grande para a satisfação dos clientes com a qualidade, com a disponibilidade, com os serviços e com a marca. Provavelmente, a norma ISO 9001 ainda é deficiente em requisitos que proporcionem às empresas uma melhor percepção da satisfação dos clientes com o preço.

Os graus de contribuição do sistema ISO 9000 nos componentes da perspectiva financeira alcançaram níveis mais baixos em comparação com as outras perspectivas. Das cinco variáveis analisadas, quatro apresentaram média abaixo de sete, considerando-se um nível de significância estatística de 0,05 . Somente o componente - valor econômico para os clientes - se comportou de forma diferenciada, não permitindo concluir sobre a sua validação. Neste caso, percebe-se que residem nesta perspectiva áreas indicadas para melhoria nas próximas revisões da norma ISO 9000, apesar de já haver uma tendência de incorporação de mais temas ligados ao desempenho no escopo das normas dessa série.

O desempenho geral do sistema ISO 9000 foi avaliado com uma média de 7,74 e desvio padrão de 1,22 , respectivamente. $O$ teste realizado demonstra a credibilidade deste sistema perante as empresas, pois o grau de contribuição desse sistema da qualidade para o desempenho geral das empresas analisadas é avaliado como grande. Já o desempenho médio do sistema ISO 9000 nas quatro perspectivas do Balanced Scorecard se comportou conforme a Figura 1. Agrupando-se as variáveis, percebe-se uma maior contribuição desse sistema nas perspectivas aprendizado e crescimento e cliente. Na perspectiva financeira há um menor impacto e na perspectiva interna, a contribuição alcançou níveis intermediários. 
Figura 1 - Contribuição do sistema ISO 9000 no desempenho estratégico

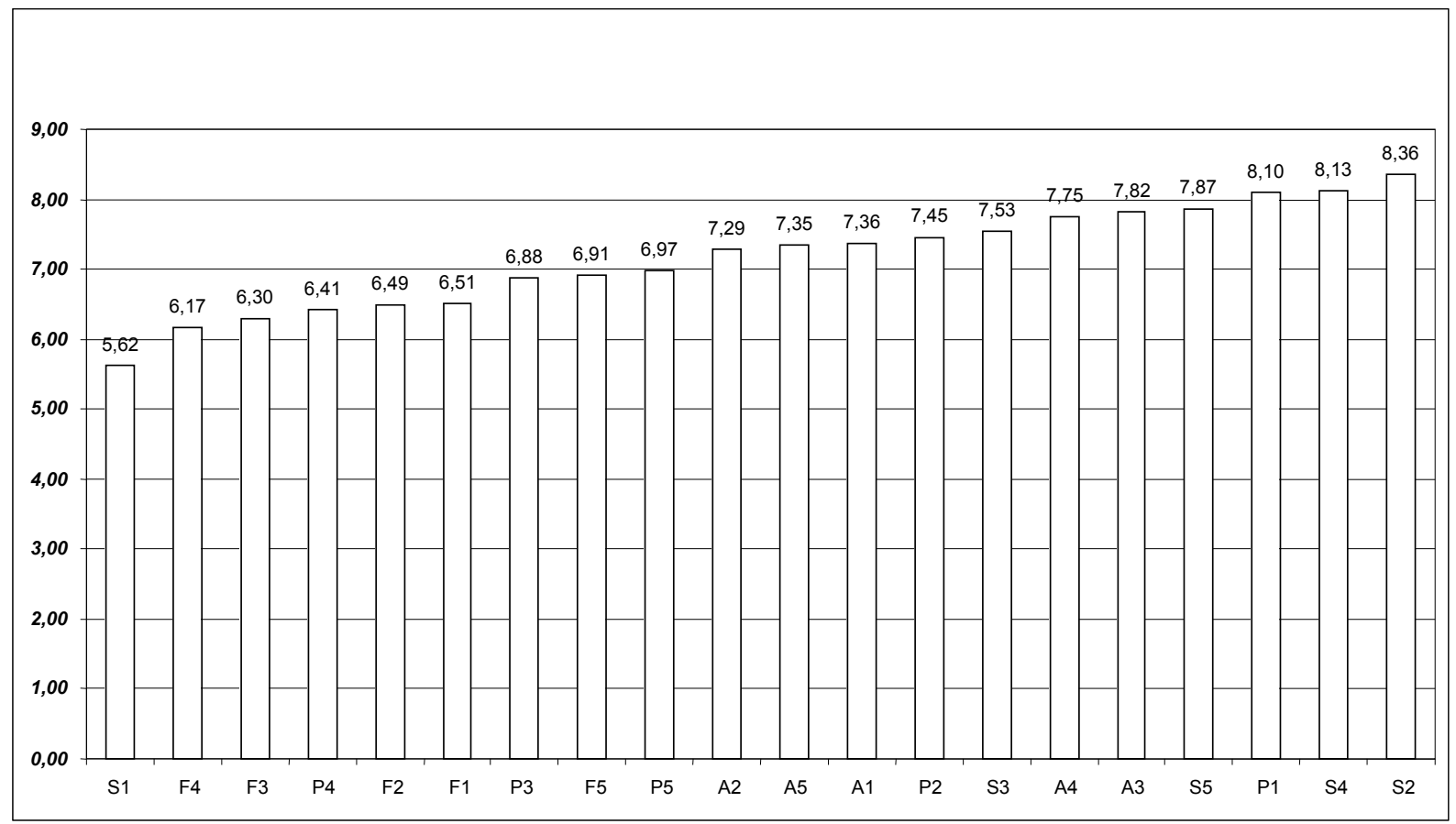

A análise das relações entre os componentes do Balanced Scorecard com o desempenho global, através da utilização da técnica estatística de regressão múltipla, é elaborada para as funções dirigentes e gerentes. Nesta etapa, procura-se explicitar quais as variáveis mais determinantes do desempenho geral e quais as contribuições relativas dos diversos componentes analisados. Para os dirigentes, cujo número total atingiu 26 entrevistados, o fator de determinação ajustado $\mathrm{R}^{2}$ alcança 0,901, através do método Backward Stepwise Regression. As variáveis mais significativas são: a satisfação dos clientes com a qualidade e os processos regulatórios e sociais, de apoio e logísticos e de produção, o que corrobora a tese de que o sistema da qualidade ISO 9000 apresenta uma contribuição significativa no desempenho estratégico. Cinco variáveis das vinte selecionadas são significativas: Satisfação dos clientes com a qualidade $(\beta=2,07)$; Processos regulatórios e sociais $(\beta=1,33)$; Processos de apoio e logísticos $(\beta=1,310)$; Processos de produção $(\beta=1,09)$ e Satisfação dos clientes com os serviços $(\beta=0,293)$. Para os dirigentes consultados, alcançar níveis elevados de desempenho global significa ter clientes satisfeitos com a qualidade e serviços e processos eficientes.

Por outro lado, na visão dos 51 gerentes consultados, a matriz de correlação entre os componentes está mostrada no Anexo 3. Com um fator de determinação $\mathrm{R}^{2}$ de 0,701 , obtido através do método Forward Stepwise Regression, as variáveis mais significativas foram: Retorno sobre o capital empregado ( $\beta=0,835)$; Utilização dos ativos da empresa $(\beta=0,476)$ e Cultura organizacional $(\beta=0,425)$. As variáveis mais significativas, que contribuem para o desempenho 
estratégico das empresas estudadas, se concentram nas perspectivas financeira e aprendizado e crescimento.

\section{Conclusões}

A contribuição das variáveis selecionadas no desempenho estratégico é vista de forma diferenciada pelos respondentes. O sistema de gestão da qualidade ISO 9000 contribui em maior grau no desempenho das variáveis relacionadas com a satisfação dos clientes com a qualidade e serviços e com os processos de produção e em menor grau com as variáveis relacionadas com o preço e com a perspectiva financeira. Este resultado é coerente com o foco principal do sistema ISO 9000, que é a satisfação do cliente. Os resultados financeiros aparecem à medida que as ações estratégicas são desenvolvidas, já que a satisfação do cliente leva à sua fidelidade e conseqüentemente, a uma maior lucratividade no futuro (GONZÁLEZ et al., 2004). De modo geral, o sistema agrega valor às organizações pesquisadas.

Por perspectiva, o sistema ISO 9000 apresenta um grau de contribuição grande nos componentes da perspectiva de aprendizado e crescimento. No âmbito da perspectiva interna, a contribuição do sistema ISO 9000 se comportou de maneira heterogênea. O sistema influencia com um grau de contribuição grande na satisfação dos clientes com a qualidade, com a disponibilidade, com os serviços e com a marca. A contribuição do sistema ISO 9000 nos componentes da perspectiva financeira alcançou níveis mais baixos em comparação com as outras perspectivas.

De acordo com a análise de regressão múltipla empreendida, as variáveis mais significativas, na visão dos dirigentes, foram concentradas nas perspectivas interna e cliente, quais sejam: processos de produção; processos regulatórios e sociais; processos de apoio e logísticos; satisfação dos clientes com a qualidade e satisfação dos clientes com os serviços. Na opinião dos gerentes, as variáveis com maior nível de significância estatística são: cultura organizacional; utilização dos ativos da empresa e retorno sobre o capital empregado.

\footnotetext{
Abstract

The objective of this article is analyzing the contribution grade of the ISO 9000 quality system to the strategic performance of the Brazilian firms in the Chemistry sector, using the Balanced Scorecard system as reference. One research is developed to get technical information about these two management systems, with emphasis in their integration. After, a quantitative research is implemented using a questionnaire, to know and to get necessaries data for improving statistical estimations of the multiple relationships between the variables and the strategic performance for the universe of 352 Brazilians firms with ISO 9000 as quality system. The size of the sample totalized 111 firms, which represent more than $31 \%$ of the selected universe. The main results show congruent visions of directors and managers about the significant variables that contribute for the strategic performance of the analyzed firms. The ISO 9000 system presents a big contribution to the strategic performance, when referred by the four perspectives of the Balanced Scorecard: learning
} 
and growth; internal process; customer and financial. Better performance was verified in the learning and growth perspective and reasonable performance was verified in the financial perspective.

Key-words: quality management, performance, ISO 9000, balanced scorecard, strategy.

\section{Referências}

ABNT. NBR ISO 9001:2000 (2000) - Sistemas de Gestão da Qualidade. Requisitos. Rio de Janeiro, 2000..

BENNER, M. \& TUSHMAN, M. Exploitation, exploration and process management: The productivity dilemma revised. Academy of Management Review, v. 28, n. 2, p. 238-256, 2003.

cross'

BRANDSTETTER, M. C. G. O. ; OLIVEIRA, E. S.; MELlO, F. F. P.; NEVES, L. A.; GARCIA, T. G. \& RORIZ, T. S. Análise do impacto do processo de implementação de sistemas de gestão da qualidade em empresas construtoras. XXII Encontro Nacional de Engenharia de Produção - Curitiba, PR, Brasil, 23 a 25 de outubro.

CAVALCANTI, R. F. V. C.; MIRANDA, L. C. \& DA SILVA, A. C. A Utilização da Certificação ISO e a Medição de Desempenho na Construção Civil. XXVIII Encontro Nacional da ANPAD, 15p, 2004.

CERQUEIRA, A. C. P. \& MORAES, W. F. A. Estratégias de qualidade e desempenho empresarial: evidências da associação. XXIII Encontro Nacional de Engenharia de Produção - Ouro Preto, MG, Brasil, 21 a 24 de outubro, 2003.

CHOW-CHUA, C.; GOH, M. \& WAN, T. B. Does ISO 9000 certification improve business performance? International Journal of Quality \& Reliability Management, v. 20, n. 8, p. 936-953, 2003.

cross ${ }^{\text {ef }}$

COLTRO, A. Efeito da globalização/regionalização: impacto modernizador do sistema de certificação ISO9000 na competitividade das organizações. XVIII Encontro Nacional de Engenharia de Produção - Niterói, RJ, Brasil, 21 a 27 de setembro, 1998.

CONCA, F. J.; LLOPIS, J. \& TARÍ, J. J. Development of a measure to assess quality management in certified firms. European Journal of Operational Research, v. 156, p. 683-697, 2004.

cross ${ }^{\text {ref }}$

COSTA, M. M. \& LORENTE, A. R. M. Comparison of TQM and ISO 9000 effects in company performance: an empirical study in spanish companies. Abstract 002-0075. Second World Conference on POM and 15th Annual POM Conference, Cancun, México, 30 de abril a 3 de maio, 2004.

DIMARA, E.; SKURAS, D. \& TSEKOURAS, K. Strategic orientation and financial performance of firms implementing ISO 9000. International Journal of Quality \& Reliability Management, v. 21, n. 1, p. 72-89, 2004.

cross $^{\text {ref }}$

GONZÁLEZ, M. O. A.; AMORIM, C. G. \& RAMOS, R. E. B. A relação entre a satisfação e a fidelidade do cliente com a lucratividade da empresa. XXIV Encontro Nacional de Engenharia de Produção, Florianópolis, SC, Brasil, 3 a 5 de novembro, 8p, 2004.

HAIR, J.; ANDERSON, R. E.; TATHAM, R. L. \& BLACK, W. C. Multivariate Data Analysis. Singapore: Pearson Education, 2003.

HERAS, I.; DICK, G. P.M. \& CASADESÚS, M. ISO 9000 registration's impact on sales and profitability: A longitudinal analysis of performance before and after accreditation. International Journal of Quality and Reliability Management, v. 19, n. 2, p. 775-791, 2002.

HOQUE, Z. Total Quality Management and the Balanced Scorecard approach: A critical analysis of their potential relationships and directions for research. Critical Perspectives on Accounting, v. 14, p. 553-566, 2003. 
JOHNSON, M. \& GUSTAFSSON, A. Improving customer satisfaction, loyalty and profit. University of Michigan Business School, management series, 214p, 2000.

KAPLAN, R. S. \& NORTON, D. P. A Estratégia em Ação: Balanced Scorecard. Rio de Janeiro: Editora Campus, 1997.

LEITE, D. C. M. \& PRANCIC, E. Os indicadores de desempenho estabelecidos pela norma ISO 9001:2000 e a sua utilização como um sistema de medição de desempenho eficaz: um estudo de caso. XXIII Encontro Nacional de Engenharia de Produção - Ouro Preto, MG, Brasil, 21 a 24 de outubro, 2003.

LIMA, M. A.M.; RESENDE, M. e HASENCLEVER, L. Quality certification and performance of Brazilian firms: An empirical study. International Journal of Production Economics, v. 66, p. 143-147, 2003.

cross ${ }^{\text {ref }}$

LORENTE, A. R. M. \& COSTA, M. M. Análisis de los posibles efectos de ISO 9000 em los resultados de empresas industriales, $21 \mathrm{p}, 2003$.

LORENTE, A. R. M. \& COSTA, M. M. ISO 9000 and TQM: substitutes or complementaries? An empirical study in idustrial companies. International Journal of Quality \& Reliability Management, v. 21, n. 3, p. 260-276, 2004.

cross ${ }^{\text {ef }}$

MENEZES, M. T \& MARTINS, R. A. ISO 9000:2000 e Balanced Scorecard: Análise das oportunidades. XXIII Encontro Nacional de Engenharia de Produção - Ouro Preto, MG, Brasil, 21 a 24 de outubro, 2003.

RAHMAN, S. A comparative study of TQM practice and organizational performance of SMEs with and without ISO 9000 certification. International Journal of Quality \& Reliability Management, v. 18, n. 1, p. 35-49, 2001.

crossef

RENZI, M. F. \& CAPPELLI, L. Integration between ISO 9000 and ISO 14000: opportunities and limits. Total Quality Management, v. 11, n. 4-6, p. S489-S856, 2000.

SCHNORR, L. A. \& KRONMEYER FILHO, O. R. A sinergia e complementaridade do Balanced Scorecard com a norma ISO 9001:2000: o caso indústria química de pequeno porte. XXIII Encontro Nacional de Engenharia de Produção - Ouro Preto, MG, Brasil, 21 a 24 de outubro, 2003.

SUN, H. Total quality management, ISO 9000 certification and performance improvement. International Journal of Quality \& Reliability, v. 17, n. 2, p. 168-179, 2000.

TERZIOVSKI, M.; POWER, D. \& SOHAL, A. S. The longitudinal effects of ISO 9000 certification process on business performance. European Journal of Operational Research, v. 146, p. 580-595, 2003.

cross $^{\text {ref }}$

TERZIOVSKI, M. \& SAMSON, D. The link between total quality management practice and organizational performance. International Journal of Quality \& Reliability Management, v. 15, n. 3, p. 226-237, 1999.

cross ref

TERZIOVSKI, M.; SAMSON, D. \& DOW, D. The business value of quality management systems certification Evidence from Australia and New Zealand. Journal of Operations Management, v. 15, p. 1-18, 1997.

cross ${ }^{\text {ref }}$

\section{Dados dos autores:}

Célio Gurgel Amorim, M.Sc.

Universidade Federal do Rio Grande do Norte

Programa de Pós-graduação em Ciência e Engenharia de Petróleo (PPGCEP)

Doutorando do Programa PPGCEP

Rua Mirabeau da Cunha Melo, 1925 apto 801, Candelária, Natal, RN. 
Telefone: (84) 32347116

e-mail:celiogurgel@digizap.com.br

Rubens Eugênio Barreto Ramos, D.Sc.

Universidade Federal do Rio Grande do Norte

Programa de Pós-Graduação em Engenharia de Produção (PEP)

Campus Universitário CEP: 59078970 - Natal (RN) Brasil

Telefone: (84) 32119239

e-mail: rubens@ct.ufrn.br

Mario Orestes Aguirre González, M.Sc.

Universidade Federal de São Carlos

Programa de Pós-Graduação em Engenharia de Produção

Doutorando

Rod. Washington Luis KM, 235, CEP - 13560-000 -; São Carlos - (SP) Brasil ;

Telefone: (16) 9206 7234; (16) 33518238 Ramal 250

e-mail: mario_gonzalez_pe@yahoo.com

Recebido para publicação em: 30/10/2006

Aceito para publicação em: 05/12/2006 